\title{
Flux compactifications and naturalness
}

\author{
Wilfried Buchmuller, ${ }^{a}$ Markus Dierigl ${ }^{b, c}$ and Emilian Dudas ${ }^{d}$ \\ ${ }^{a}$ Deutsches Elektronen-Synchrotron DESY, \\ 22607 Hamburg, Germany \\ ${ }^{b}$ Institute for Theoretical Physics, Utrecht University, \\ 3584 CC Utrecht, The Netherlands \\ ${ }^{c}$ Institute of Physics, University of Amsterdam, \\ 1098 XH Amsterdam, The Netherlands \\ ${ }^{d}$ Centre de Physique Théorique, École Polytechnique, CNRS, Université Paris-Saclay, \\ F-91128 Palaiseau, France \\ E-mail: wilfried.buchmueller@desy.de,m.j.dierigl@uu.de, \\ emilian.dudas@cpht.polytechnique.fr
}

ABSTRACT: Free massless scalars have a shift symmetry. This is usually broken by gauge and Yukawa interactions, such that quantum corrections induce a quadratically divergent mass term. In the Standard Model this leads to the hierarchy problem of the electroweak theory, the question why the Higgs mass is so much smaller than the Planck mass. We present an example where a large scalar mass term is avoided by coupling the scalar to an infinite tower of massive states which are obtained from a six-dimensional theory compactified on a torus with magnetic flux. The series of divergent quantum corrections adds up to zero, and we show explicitly that the shift symmetry of the scalar is preserved in the effective four-dimensional theory despite the presence of gauge and Yukawa interaction terms.

KEywords: Effective Field Theories, Flux compactifications

ARXIV EPRINT: 1804.07497 


\section{Contents}

1 Introduction 1

2 Flux compactification on a torus 2

3 Quantum corrections and shift symmetry 6

4 Summary and outlook $\quad 12$

$\begin{array}{ll}\text { A Quantum mechanics on the magnetized torus } & 13\end{array}$

\section{Introduction}

Compactifications on tori with magnetic flux play an important role in string theories and higher-dimensional field theories (see, for example, [1-3]). Due to the index theorem they lead to a multiplicity of chiral fermions, which can be used to explain the number of quark-lepton generations [4]. Moreover, magnetic flux is an important source of supersymmetry breaking [5]. Magnetic compactifications of higher-dimensional field theories have been thoroughly studied in ref. [6]. These results have been used to construct interesting supersymmetric models of particle physics and to compute Yukawa couplings (see, for example, [6-8]). Making use of flux configurations that break supersymmetry one can also construct extensions of the Standard Model with high-scale supersymmetry [9].

The components of higher-dimensional gauge fields along compact dimensions play a special role for compact spaces with non-trivial topology. Their zero modes, often called Wilson-line (WL) scalars are interesting candidates for Higgs fields in four dimensions (4d) [10-12]. Compactifying a five-dimensional (5d) theory on a circle, or a six-dimensional (6d) theory on a torus, one finds a discrete set of large gauge transformations in the $4 \mathrm{~d}$ theory, due to the higher-dimensional gauge invariance and the non-trivial topology of the compact manifold. These large gauge transformations act as discrete shifts on WL scalars and can therefore protect their masses from quadratic divergencies. Identifying Higgs fields as WL scalars, one obtains finite Higgs masses, determined by size of the extra dimensions, $m_{H}^{2} \propto L^{-2}$, where $L$ is a typical length scale of the internal space [13-15]. This mechanism to protect Higgs masses is of interest in scenarios with large extra dimensions, where the scale of electroweak symmetry breaking is tied to the size of the compact space.

How can one protect scalar masses if the ultraviolet cutoff of the theory lies much above the scale of electroweak symmetry breaking? In this case the only known candidate for a protection mechanism is a continuous shift symmetry, like the Peccei-Quinn symmetry [16] in axion physics or the shift symmetry of a Goldstone boson in composite Higgs models (see, for example, [17]). Such a mechanism would be needed in models of high-scale supersymmetry, like the one considered in [9]. In the following we shall give an example that 
illustrates how such a shift symmetry can indeed arise in compactifications with magnetic flux, and we shall identify the higher-dimensional origin of the symmetry.

In ref. [18] we have worked out the magnetic compactification of a supersymmetric 6d Abelian gauge theory on a torus, and we have compared the results with the standard compactification without flux. In the latter case bosonic and fermionic one-loop corrections to the mass of the WL scalar are separately finite and cancel each other due to unbroken supersymmetry. On the contrary, in the case of flux compactification, bosonic and fermionic contributions are zero separately, once the complete tower of massive states is taken into account. We argued that this surprizing cancellation of an infinite number of terms is a consequence of a $6 \mathrm{~d}$ symmetry, the invariance under translations on the torus under which the WL scalar transforms with a shift. The vanishing of the one-loop corrections to the mass of the WL scalar has subsequently been carefully studied in [19].

Notice that our field theory setup was widely studied in string theory compactifications with internal magnetic fields [5, 20] and in the T-dual version of D-branes at angles (or intersecting brane models) [21-23], as a way to partially or completely break supersymmetry and to induce fermion chirality. However, a field theory approach has its own advantages, namely, more flexibility in searching for realistic models of particle physics and the avoidance of technical difficulties with quantum corrections for string theory models with broken supersymmetry, see e.g. [24].

In this paper we study the cancellation of loop corrections to the mass of the WL scalar in more detail. Since the cancellation is independent of supersymmetry, we focus on the simplest possible model, a single 6d Weyl fermion interacting with an Abelian gauge field. In section 2 we provide details of the flux compactification on a torus with emphasis on the symmetries of the $6 \mathrm{~d}$ theory and the couplings of the tower of massive states in the effective $4 \mathrm{~d}$ theory. Quantum corrections to the mass of the WL scalar are discussed in section 3. We first recall the cancellations at one-loop order once the tower of massive states is taken into account. We then show that the $4 \mathrm{~d}$ action possesses an exact shift symmetry, including the couplings to all massive states. In section 4, we summarize our results and discuss the prospects to extend the presented model to chiral Higgs models. The connection between the considered field theory and quantum mechanics on a magnetized torus is discussed in the appendix.

\section{Flux compactification on a torus}

Let us now consider a left-handed 6d Weyl fermion interacting with an Abelian gauge field, ${ }^{1}$

$$
S_{6}=\int d^{6} x\left(-\frac{1}{4} F^{M N} F_{M N}+i \bar{\Psi} \Gamma^{M} D_{M} \Psi\right)
$$

where $D_{M}=\partial_{M}+i q A_{M}, M=0, \ldots 6, F_{M N}=\partial_{M} A_{N}-\partial_{N} A_{M}$ and $\Gamma_{7} \Psi=-\Psi$. The $6 \mathrm{~d}$ space is a product of $4 \mathrm{~d}$ Minkowski space and a square torus $T^{2}$ of area $L^{2}$. It is convenient

\footnotetext{
${ }^{1}$ In the following we ignore anomalies. Note that our discussion will not change for an anomaly-free fermion spectrum.
} 
to decompose the $6 \mathrm{~d}$ Weyl spinor into two independent two-component Weyl spinors $\psi$ and $\chi$. For gamma matrices in the Weyl basis, one has ${ }^{2}$

$$
\begin{aligned}
& \Psi=\left(\begin{array}{c}
\psi_{L} \\
\psi_{R}
\end{array}\right): \quad \gamma_{5} \psi_{L}=-\psi_{L}, \quad \gamma_{5} \psi_{R}=\psi_{R}, \\
& \psi_{L}=\left(\begin{array}{c}
\psi \\
0
\end{array}\right), \quad \psi_{R}=\left(\begin{array}{c}
0 \\
\bar{\chi}
\end{array}\right) .
\end{aligned}
$$

The Weyl fermions $\psi$ and $\chi$ have charges $q$ and $-q$, respectively, and the fermionic part of the action (2.1) reads

$$
\begin{aligned}
S_{6 f}= & \int d^{6} x\left(-i \psi \sigma^{\mu} \bar{D}_{\mu} \bar{\psi}-i \chi \sigma^{\mu} D_{\mu} \bar{\chi}\right. \\
& \left.-\chi\left(\partial_{z}+\sqrt{2} q \phi\right) \psi-\bar{\chi}\left(\partial_{\bar{z}}+\sqrt{2} q \bar{\phi}\right) \bar{\psi}\right),
\end{aligned}
$$

where $D_{\mu}=\partial_{\mu}+i q A_{\mu}, \bar{D}_{\mu}=\partial_{\mu}-i q A_{\mu}$ and

$$
\phi=\frac{1}{\sqrt{2}}\left(A_{6}+i A_{5}\right), \quad z=\frac{1}{2}\left(x_{5}+i x_{6}\right), \quad \partial_{z}=\partial_{5}-i \partial_{6} .
$$

The coordinates take values in the interval $x_{5,6} \in[0, L)$. In the following we set $L=1$. The gauge kinetic term can be expressed in terms of the fields $A_{\mu}$ and $\phi$,

$$
\begin{aligned}
S_{6 g}= & \int d^{6} x\left(-\frac{1}{4} F^{M N} F_{M N}\right) \\
= & \int d^{6} x\left(-\frac{1}{4} F^{\mu \nu} F_{\mu \nu}-\partial^{\mu} \bar{\phi} \partial_{\mu} \phi-\frac{1}{4}\left(\partial_{z} \bar{\phi}+\partial_{\bar{z}} \phi\right)^{2}\right. \\
& \left.-\frac{1}{2} \partial_{\bar{z}} A^{\mu} \partial_{z} A_{\mu}-\frac{i}{\sqrt{2}} \partial_{\mu} A^{\mu}\left(\partial_{z} \bar{\phi}-\partial_{\bar{z}} \phi\right)\right) .
\end{aligned}
$$

Constant magnetic flux in the compact dimensions corresponds to a vacuum configuration. For $\left\langle A_{5}\right\rangle=-\frac{1}{2} f x_{6},\left\langle A_{6}\right\rangle=\frac{1}{2} f x_{5}$, corresponding to $\langle\phi\rangle=\frac{1}{\sqrt{2}} f \bar{z}$, the vacuum field equations are satisfied, ${ }^{3}$

$$
\partial_{z}\left(\partial_{z}\langle\bar{\phi}\rangle+\partial_{\bar{z}}\langle\phi\rangle\right)=0
$$

The magnetic flux is quantized in units of the torus area,

$$
\frac{q}{2 \pi} \int_{T^{2}} F=\frac{q}{2 \pi} f=N \in \mathbb{Z}
$$

Shifting the scalar field $\phi$ around the flux background,

$$
\phi=\frac{f}{\sqrt{2}} \bar{z}+\varphi
$$

\footnotetext{
${ }^{2}$ We follow the conventions in ref. [25]. Our $6 \mathrm{~d}$ gamma matrices satisfy the algebra $\left\{\Gamma_{M}, \Gamma_{N}\right\}=-2 \eta_{M N}$, with $\operatorname{diag}\left(\eta_{M N}\right)=(-1,+1, \ldots,+1)$.

${ }^{3}$ Note that this non-trivial gauge background requires the introduction of four patches on the torus.
} 
the $6 \mathrm{~d}$ action takes the form of eqs. (2.3) and (2.5), with $\phi$ replaced by $\varphi$, up to a cosmological constant ${ }^{4}$ and a flux-dependent bilinear term of the Weyl fermions,

$$
\begin{aligned}
S_{6}= & \int d^{6} x\left(-\frac{1}{4} F^{\mu \nu} F_{\mu \nu}-\partial^{\mu} \bar{\varphi} \partial_{\mu} \varphi-\frac{1}{4}\left(\partial_{z} \bar{\varphi}+\partial_{\bar{z} \varphi}\right)^{2}-\frac{1}{2} f^{2}\right. \\
& -\frac{1}{2} \partial_{\bar{z}} A^{\mu} \partial_{z} A_{\mu}-\frac{i}{\sqrt{2}} \partial_{\mu} A^{\mu}\left(\partial_{z} \bar{\varphi}-\partial_{\bar{z}} \varphi\right) \\
& -i \psi \sigma^{\mu} \bar{D}_{\mu} \bar{\psi}-i \chi \sigma^{\mu} D_{\mu} \bar{\chi} \\
& \left.-\chi\left(\partial_{z}+q f \bar{z}+\sqrt{2} q \varphi\right) \psi-\bar{\chi}\left(\partial_{\bar{z}}+q f z+\sqrt{2} q \bar{\varphi}\right) \bar{\psi}\right) .
\end{aligned}
$$

The action is invariant under translations on the torus, which act in the standard way as $\delta_{T}=\epsilon \partial_{z}+\bar{\epsilon} \partial_{\bar{z}}$ on the fields $A_{\mu}, \psi$ and $\chi$. The breaking of translational invariance by the background gauge field can be compensated by a shift of $\varphi$,

$$
\delta_{T} \varphi=\left(\epsilon \partial_{z}+\bar{\epsilon} \partial_{\bar{z}}\right) \varphi+\frac{\bar{\epsilon}}{\sqrt{2}} f .
$$

The Lagrangian density in (2.9) then transforms into a total divergence. ${ }^{5}$ Furthermore, the action is invariant with respect to the following local $6 \mathrm{~d}$ transformation,

$$
\varphi_{\Lambda}=\varphi-\frac{1}{\sqrt{2}} \partial_{z} \Lambda, \quad \psi_{\Lambda}=e^{q \Lambda} \psi, \quad \chi_{\Lambda}=e^{-q \Lambda} \chi, \quad \Lambda=f(\alpha \bar{z}-\bar{\alpha} z),
$$

where $\alpha$ is a complex parameter. Such transformations have first been considered in [26]. Note that they change the boundary conditions of the fermion wave functions. For infinitesimal $\alpha$ the transformation reads

$$
\delta_{\Lambda} \varphi=-\frac{1}{\sqrt{2}} \partial_{z} \Lambda, \quad \delta_{\Lambda} \psi=q \Lambda \psi, \quad \delta_{\Lambda} \chi=-q \Lambda \chi .
$$

For the complex scalar field, the gauge transformation corresponds to a shift,

$$
\delta_{\Lambda} \varphi=\frac{\bar{\alpha}}{\sqrt{2}} f .
$$

In order to obtain the effective $4 \mathrm{~d}$ action one expands the $6 \mathrm{~d}$ fields into mode functions corresponding to eigenstates of the kinetic term of the compact dimensions. For charged fields these are Landau levels obtained from an harmonic oscillator algebra [5, 15, 27]. The identification of annihilation and creation operators depends on the sign of $q f$. Without loss of generality we choose $q f>0$. There are two pairs of annihilation and creation operators

$$
\begin{array}{ll}
a_{+}=\frac{i}{\sqrt{2 q f}}\left(\partial_{z}+q f \bar{z}\right), & a_{+}^{\dagger}=\frac{i}{\sqrt{2 q f}}\left(\partial_{\bar{z}}-q f z\right), \\
a_{-}=\frac{i}{\sqrt{2 q f}}\left(\partial_{\bar{z}}+q f z\right), & a_{-}^{\dagger}=\frac{i}{\sqrt{2 q f}}\left(\partial_{z}-q f \bar{z}\right) .
\end{array}
$$

\footnotetext{
${ }^{4}$ Once gravity is included, the backreaction of the cosmological term on the compact manifold has to be taken into account. However, we have applications in mind with $f \ll M_{\mathrm{PL}}$, where gravitational corrections are expected to be small.

${ }^{5}$ To prove the invariance of the action one has to take into account that gauge field and charged fermions are fiber bundles defined on four patches of the torus.
} 
They satisfy the commutation relations $\left[a_{ \pm}, a_{ \pm}^{\dagger}\right]=1,\left[a_{ \pm}, a_{\mp}\right]=0,\left[a_{ \pm}, a_{\mp}^{\dagger}\right]=0$. In terms of the annihilation and creation operators the mass-square operators for the fermions $\psi$ with charge $+q$ and $\chi$ with charge $-q$ are given by

$$
\mathcal{M}_{+}^{2}=2 q f a_{+}^{\dagger} a_{+}, \quad \mathcal{M}_{-}^{2}=2 q f\left(a_{-}^{\dagger} a_{-}+1\right) .
$$

The ground state wave functions are determined by

$$
a_{+} \xi_{0, j}=0, \quad a_{-} \bar{\xi}_{0, j}=0,
$$

where $j=0, \ldots|N|-1$ labels the degeneracy of the ground state. An orthonormal set of higher mode functions is given by

$$
\xi_{n, j}=\frac{i^{n}}{\sqrt{n !}}\left(a_{+}^{\dagger}\right)^{n} \xi_{0, j}, \quad \bar{\xi}_{n, j}=\frac{i^{n}}{\sqrt{n !}}\left(a_{-}^{\dagger}\right)^{n} \bar{\xi}_{0, j} .
$$

Annihilation and creation operators act on these mode functions as

$$
\begin{array}{ll}
a_{+} \xi_{n, j}=i \sqrt{n} \xi_{n-1, j}, & a_{+}^{\dagger} \xi_{n, j}=-i \sqrt{n+1} \xi_{n+1, j}, \\
a_{-} \bar{\xi}_{n, j}=i \sqrt{n} \bar{\xi}_{n-1, j}, & a_{-}^{\dagger} \bar{\xi}_{n, j}=-i \sqrt{n+1} \bar{\xi}_{n+1, j},
\end{array}
$$

and the mode expansions of the fermion fields $\psi$ and $\chi$ with charges $+q$ and $-q$, respectively, read

$$
\psi=\sum_{n, j} \psi_{n, j} \xi_{n, j}, \quad \chi=\sum_{n, j} \chi_{n, j} \bar{\xi}_{n, j} .
$$

Since the gauge fields $A_{\mu}$ and $\varphi$ do not feel the flux, they have an expansion in terms of standard Kaluza-Klein modes. The theory has a number of $4 \mathrm{~d}$ zero modes. According to eq. (2.16), and in accord with the index theorem, there are $|N|$ left-handed fermionic zero modes $\psi_{0, j}$. Moreover, there will be zero modes $A_{0 \mu}$ due to $4 \mathrm{~d}$ gauge invariance, and, up to quantum corrections, a massless complex scalar $\varphi_{0} .{ }^{6}$ The action for the zero mode $\varphi_{0}$, $A_{0 \mu}$, and the matter fields is easily obtained by inserting the expansions (2.21) into the action (2.9). The result reads

$$
\begin{aligned}
S_{4}= & \int d^{4} x\left(-\partial^{\mu} \bar{\varphi}_{0} \partial_{\mu} \varphi_{0}+\sum_{n, j}\left(-i \psi_{n, j} \sigma^{\mu} \bar{D}_{\mu} \bar{\psi}_{n, j}-i \chi_{n, j} \sigma^{\mu} D_{\mu} \bar{\chi}_{n, j}\right.\right. \\
& \left.\left.-\sqrt{2 q f(n+1)} \chi_{n, j} \psi_{n+1, j}-\sqrt{2} q \varphi_{0} \chi_{n, j} \psi_{n, j}+\text { h.c. }\right)\right) .
\end{aligned}
$$

This is the fermionic part of the supersymmetric action derived in [18]. It describes $|N|$ left-handed fermions $\psi_{L j}$ and an infinite tower of massive Dirac fermions $\Psi_{n, j}$,

$$
\psi_{L j}=\left(\begin{array}{c}
\psi_{0, j} \\
0
\end{array}\right), \quad \Psi_{n, j}=\left(\begin{array}{c}
\psi_{n+1, j} \\
\bar{\chi}_{n, j}
\end{array}\right),
$$

\footnotetext{
${ }^{6}$ Note that in a supergravity extension of the present model the zero modes $A_{0 \mu}$ become massive due to the Stueckelberg mechanism. For a recent discussion of the interplay of flux and the Green-Schwarz mechanism, see [28].
} 
which interact via Yukawa couplings with a massless scalar,

$$
\begin{aligned}
S_{4}= & \int d^{4} x\left(-\partial^{\mu} \bar{\varphi}_{0} \partial_{\mu} \varphi_{0}+\sum_{n, j}\left(i \bar{\psi}_{L j} \gamma^{\mu} D_{\mu} \psi_{L j}+i \bar{\Psi}_{n, j} \gamma^{\mu} D_{\mu} \Psi_{n, j}\right.\right. \\
& +\sqrt{2 q f(n+1)} \bar{\Psi}_{n, j} \Psi_{n, j}+\sqrt{2} q \varphi_{0}\left(\bar{\Psi}_{0, j} \frac{1-\gamma_{5}}{2} \psi_{L j}+\bar{\Psi}_{n+1, j} \frac{1-\gamma_{5}}{2} \Psi_{n, j}\right) \\
& \left.\left.+\sqrt{2} q \bar{\varphi}_{0}\left(\bar{\psi}_{L j} \frac{1+\gamma_{5}}{2} \Psi_{0, j}+\bar{\Psi}_{n, j} \frac{1+\gamma_{5}}{2} \Psi_{n+1, j}\right)\right)\right) .
\end{aligned}
$$

Note that the scalar $\varphi_{0}$ couples to different mass eigenstates. Integrating out the heavy fermions $\Psi_{n, j}$ yields the effective low energy action of the zero modes $\varphi_{0}$ and $\psi_{L j}$. The first contribution is due to the exchange of $\Psi_{0, j}$. Expanding the propagator as

$$
\left\langle\Psi_{0, j}(x) \bar{\Psi}_{0, j^{\prime}}\left(x^{\prime}\right)\right\rangle=-\frac{i}{m_{0}}\left(1+\frac{i \gamma^{\mu} \partial_{\mu}}{m_{0}}+\ldots\right) \delta_{j, j^{\prime}} \delta^{4}\left(x-x^{\prime}\right),
$$

with $m_{0}=\sqrt{2 q f}$, one obtains

$$
\begin{aligned}
S_{\mathrm{eff}}= & \int d^{4} x\left(-\partial^{\mu} \bar{\varphi}_{0} \partial_{\mu} \varphi_{0}+i \bar{\psi}_{L j} \gamma^{\mu} D_{\mu} \psi_{L j}\right. \\
& \left.+i \frac{q^{2}}{m_{0}^{2}} \bar{\psi}_{L, j} \gamma^{\mu} \psi_{L, j}\left(\bar{\varphi}_{0} \partial_{\mu} \varphi_{0}-\partial_{\mu} \bar{\varphi}_{0} \varphi_{0}\right)+\ldots\right),
\end{aligned}
$$

where we have used the equation of motion for $\psi_{L j}$ to leading order, i.e. $\gamma^{\mu} \partial_{\mu} \psi_{L j}=0$. One easily verifies that this effective action is invariant under a constant shift of $\varphi_{0}$.

The effective action (2.26) is very different from the $4 \mathrm{~d}$ action without magnetic flux. In this case one obtains a vector-like theory, and after spontaneous symmetry breaking the lowest states of the spectrum consist of a Dirac fermion, a real scalar and a vector, which all have masses of the order of the compactification scale. No massless states are left. On the contrary, the action (2.26) does contain massless chiral fermions and a WL scalar which is kept massless by a continuous shift symmetry. However, contrary to the case of the Standard Model, its vacuum expectation value does not give mass to the chiral fermions.

\section{Quantum corrections and shift symmetry}

In general, Yukawa interactions violate the shift symmetry of a free massless scalar, and as a consequence quantum corrections generate a mass term. Indeed, keeping the lightest massive fermion $\Psi_{0, j}$ in addition to the zero modes $\Psi_{L j}$, one obtains from the standard one-loop diagrams (see figure 1, left),

$$
\begin{aligned}
\delta m_{\varphi_{0}}^{2} & =-2 q^{2}|N| \int \frac{d^{4} k}{(2 \pi)^{4}} \frac{2 k^{2}}{k^{2}\left(k^{2}+2 q f\right)} \\
& =-\frac{q^{2}|N|}{4 \pi^{2}}\left(\Lambda^{2}-2 q f \ln \left(\frac{\Lambda^{2}}{2 q f}\right)+\ldots\right),
\end{aligned}
$$



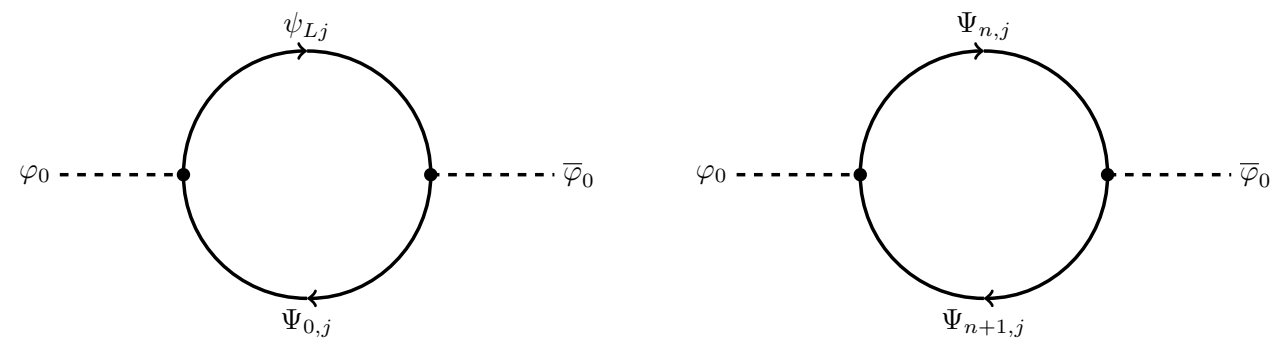

Figure 1. One-loop contributions to the scalar mass term. Left: contribution of $\psi_{L j}$ and $\Psi_{0, j}$; right: contributions of the massive fermions $\Psi_{n, j}$ and $\Psi_{n+1, j}$.

where we have introduced a momentum cutoff $\Lambda$ as regulator. Usually, the quadratic divergence is removed by a counter term, leaving an undetermined finite mass for the scalar $\varphi_{0}$. In ref. [18] it was shown that the situation drastically changes once the Yukawa couplings to the entire tower of massive states are taken into account (see figure 1, right). One then obtains

$$
\begin{aligned}
\delta m_{\varphi_{0}}^{2} & =-2 q^{2}|N| \sum_{n} \int \frac{d^{4} k}{(2 \pi)^{4}} \frac{2 k^{2}}{\left(k^{2}+2 q f n\right)\left(k^{2}+2 q f(n+1)\right)} \\
& =4 q^{2}|N| \sum_{n} \int \frac{d^{4} k}{(2 \pi)^{4}}\left(\frac{n}{k^{2}+2 q f n}-\frac{n+1}{k^{2}+2 q f(n+1)}\right) .
\end{aligned}
$$

Using the Schwinger representation of the propagators, performing the momentum integrations and interchanging $t$-integration and summation, one finds

$$
\begin{aligned}
\delta m_{\varphi_{0}}^{2} & =\frac{q^{2}}{4 \pi^{2}}|N| \sum_{n} \int_{0}^{\infty} d t \frac{1}{t^{2}}\left(n e^{-2 q f n t}-(n+1) e^{-2 q f(n+1) t}\right) \\
& =\frac{q^{2}}{4 \pi^{2}}|N| \int_{0}^{\infty} d t \frac{1}{t^{2}}\left(\frac{e^{2 q f t}}{\left(e^{2 q f t}-1\right)^{2}}-\frac{e^{2 q f t}}{\left(e^{2 q f t}-1\right)^{2}}\right) \\
& =0 .
\end{aligned}
$$

To obtain this remarkable cancellation it is crucial to perform the summation before the momentum integration, as in ref. [14]. In this way the symmetries of the gauge theory in the compact dimensions are preserved.

What is the origin of the cancellation of the quantum corrections to the scalar mass term and can one understand it at the level of the four-dimensional theory? As discussed in the previous section the six-dimensional theory is invariant under translations, which include a shift of the scalar field $\varphi_{0}$. The generators of the translations, $\partial_{z}$ and $\partial_{\bar{z}}$, do not commute with the mass-squared operators $\mathcal{M}_{ \pm}^{2}$. However, the mode functions are eigenfunctions of $\mathcal{M}_{ \pm}^{2}$. Therefore, they have no simple transformation law under the action of $\partial_{z}$ and $\partial_{\bar{z}}$. Instead, the whole tower is reshuffled. A simple transformation of the mode function can be obtained by combining translations with the transformation $\delta_{\Lambda}$, eq. (2.12), 
as follows:

$$
\begin{aligned}
\delta \psi & =\left(\delta_{T}+\delta_{\Lambda, \alpha=\epsilon}\right) \psi \\
& =\left(\epsilon \partial_{z}+\bar{\epsilon} \partial_{\bar{z}}+q f(\epsilon \bar{z}-\bar{\epsilon} z)\right) \psi \\
& =-i \sqrt{2 q f}\left(\epsilon a_{+}+\bar{\epsilon} a_{+}^{\dagger}\right) \psi .
\end{aligned}
$$

Clearly, this infinitesimal transformation only connects mode functions of neighboring mass eigenvalues. As we show in appendix A, this symmetry also manifests itself in the quantum mechanical analysis of a charged particle on a magnetized torus. Using eqs. (2.19) one obtains

$$
\begin{aligned}
\delta \psi & =-i \sqrt{2 q f} \sum_{n, j} \psi_{n, j}\left(\epsilon a_{+}+\bar{\epsilon} a_{+}^{\dagger}\right) \xi_{n, j}=\sum_{n, j} \delta \psi_{n, j} \xi_{n, j}, \\
\delta \psi_{n, j} & =\sqrt{2 q f}\left(\epsilon \sqrt{n+1} \psi_{n+1, j}-\bar{\epsilon} \sqrt{n} \psi_{n-1, j}\right) .
\end{aligned}
$$

Analogously, the transformation of the matter field $\chi$ with charge $-q$ is given by

$$
\begin{aligned}
\delta \chi & =\left(\delta_{T}+\delta_{\Lambda, \alpha=\epsilon}\right) \chi \\
& =\left(\epsilon \partial_{z}+\bar{\epsilon} \partial_{\bar{z}}-q f(\epsilon \bar{z}-\bar{\epsilon} z)\right) \chi \\
& =-i \sqrt{2 q f}\left(\epsilon a_{-}^{\dagger}+\bar{\epsilon} a_{-}\right) \chi .
\end{aligned}
$$

Using eqs. (2.20) one finds

$$
\begin{aligned}
\delta \chi & =-i \sqrt{2 q f} \sum_{n, j} \chi_{n, j}\left(\epsilon a_{-}^{\dagger}+\bar{\epsilon} a_{-}\right) \bar{\xi}_{n, j}=\sum_{n, j} \delta \chi_{n, j} \bar{\xi}_{n, j}, \\
\delta \chi_{n, j} & =\sqrt{2 q f}\left(-\epsilon \sqrt{n} \chi_{n-1, j}+\bar{\epsilon} \sqrt{n+1} \chi_{n+1, j}\right) .
\end{aligned}
$$

Given the transformation laws (3.5) and (3.7) it is straightforward to verify the invariance of the action (2.22). For instance, for the Yukawa term one has

$$
\begin{aligned}
\delta\left(\sum_{n, j} \chi_{n, j} \psi_{n, j}\right)= & \sqrt{2 q f} \sum_{n, j}\left(-\epsilon \sqrt{n} \chi_{n-1, j} \psi_{n, j}+\bar{\epsilon} \sqrt{n+1} \chi_{n+1, j} \psi_{n, j}\right. \\
& \left.+\epsilon \sqrt{n+1} \chi_{n, j} \psi_{n+1, j}-\bar{\epsilon} \sqrt{n} \chi_{n, j} \psi_{n-1, j}\right)=0 .
\end{aligned}
$$

Similarly, also the fermion kinetic terms are invariant. The remaining part is

$$
\begin{aligned}
\delta S_{4} \supset & \int d^{4} x\left(-\partial^{\mu} \delta \bar{\varphi}_{0} \partial_{\mu} \varphi_{0}-\partial^{\mu} \bar{\varphi}_{0} \partial_{\mu} \delta \varphi_{0}\right. \\
& \left.+\sum_{n, j}\left(-\sqrt{2 q f(n+1)} \delta\left(\chi_{n, j} \psi_{n+1, j}\right)-\sqrt{2} q \delta \varphi_{0} \chi_{n, j} \psi_{n, j}+\text { h.c. }\right)\right) .
\end{aligned}
$$

The variation of the mass term reads

$$
\begin{aligned}
& \delta\left(\sum_{n, j} \sqrt{n+1} \chi_{n, j} \psi_{n+1, j}\right)=\sqrt{2 q f} \sum_{n, j} \sqrt{n+1}\left(-\epsilon \sqrt{n} \chi_{n-1, j} \psi_{n+1, j}\right. \\
&\left.+\bar{\epsilon} \sqrt{n+1} \chi_{n+1, j} \psi_{n+1, j}+\epsilon \sqrt{n+2} \chi_{n, j} \psi_{n+2, j}-\bar{\epsilon} \sqrt{n+1} \chi_{n, j} \psi_{n, j}\right) \\
&=-\bar{\epsilon} \sqrt{2 q f} \sum_{n, j} \chi_{n, j} \psi_{n, j}
\end{aligned}
$$



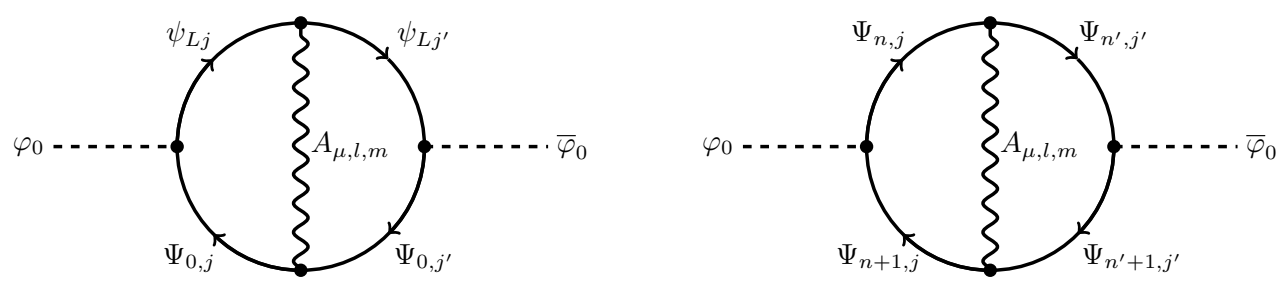

Figure 2. Two-loop contributions to the scalar mass term which involve Kaluza-Klein modes of the vector field.

which yields for the $4 \mathrm{~d}$ action

$$
\begin{aligned}
\delta S_{4} \supset & \int d^{4} x\left(-\partial^{\mu} \delta \bar{\varphi}_{0} \partial_{\mu} \varphi_{0}-\partial^{\mu} \bar{\varphi}_{0} \partial_{\mu} \delta \varphi_{0}\right. \\
& \left.+\left(2 q f \bar{\epsilon}-\sqrt{2} q \delta \varphi_{0}\right) \sum_{n, j} \chi_{n, j} \psi_{n, j}+\text { h.c. }\right)
\end{aligned}
$$

Hence, the action is invariant if the scalar $\varphi_{0}$ transforms as

$$
\delta \varphi_{0}=\sqrt{2} \bar{\epsilon} f .
$$

This is precisely the shift inferred from the two transformation laws of the $6 \mathrm{~d}$ field $\varphi$ given in eqs. (2.10) and (2.13).

So far we have discussed the coupling of the zero mode $\varphi_{0}$ to the matter fields $\psi_{n, j}$ and $\chi_{n, j}$, and we have seen that at one-loop order no mass term is generated. However, the full theory also contains the Kaluza-Klein excitations of the gauge fields $A_{\mu}$ and $\varphi$, which enter at higher loop order (see figure 2), and it is an important question whether also these corrections preserve the shift symmetry of $\varphi_{0}$. Splitting the gauge fields into zero modes and KK excitations, one has

$$
\begin{aligned}
\varphi & =\varphi_{0}+\varphi^{\prime}, & A_{\mu} & =A_{0 \mu}+A_{\mu}^{\prime}, \\
\varphi^{\prime} & =\sum_{l, m} \varphi_{l, m} \lambda_{l, m}, & A_{\mu}^{\prime} & =\sum_{l, m} A_{\mu, l, m} \lambda_{l, m},
\end{aligned}
$$

with zero modes $(l=m=0)$ excluded in the sum, and with the standard orthonormal mode functions

$$
\lambda_{l, m}=e^{z M_{l, m}-\bar{z} \bar{M}_{l, m}}=\bar{\lambda}_{-l,-m}, \quad M_{l, m}=2 \pi(m+i l) .
$$

Since the vector field is real, one has $A_{\mu, l, m}=A_{\mu,-l,-m}$. For the gauge fields the masssquared operator is given by $\mathcal{M}^{2}=-\partial_{\bar{z}} \partial_{z}$, which commutes with the generators of translations. In fact, they are eigenfunctions of $\partial_{\bar{z}}$ and $\partial_{z}$. Hence, a simple transformation law is obtained for the transformation $\delta$,

$$
\begin{gathered}
\delta \varphi^{\prime}=\left(\epsilon \partial_{z}+\bar{\epsilon} \partial_{\bar{z}}\right) \varphi^{\prime}=\sum_{l, m} \delta \varphi_{l, m} \lambda_{l, m} \\
\delta A_{\mu}^{\prime}=\left(\epsilon \partial_{z}+\bar{\epsilon} \partial_{\bar{z}}\right) A_{\mu}^{\prime}=\sum_{l, m} \delta A_{\mu, l, m} \lambda_{l, m}
\end{gathered}
$$


which yields the transformation law of the mode functions

$$
\begin{aligned}
\delta \varphi_{l, m} & =\left(\epsilon M_{l, m}-\bar{\epsilon} \bar{M}_{l, m}\right) \varphi_{l, m}, \\
\delta A_{\mu, l, m} & =\left(\epsilon M_{l, m}-\bar{\epsilon} \bar{M}_{l, m}\right) A_{\mu, l, m} .
\end{aligned}
$$

These equations together with eqs. (3.5), (3.7) and (3.12),

$$
\begin{aligned}
\delta \varphi_{0} & =\sqrt{2} \bar{\epsilon} f \\
\delta \psi_{n, j} & =\sqrt{2 q f}\left(\epsilon \sqrt{n+1} \psi_{n+1, j}-\bar{\epsilon} \sqrt{n} \psi_{n-1, j}\right), \\
\delta \chi_{n, j} & =\sqrt{2 q f}\left(-\epsilon \sqrt{n} \chi_{n-1, j}+\bar{\epsilon} \sqrt{n+1} \chi_{n+1, j}\right),
\end{aligned}
$$

define the transformation behavior of all $4 \mathrm{~d}$ fields.

Given the mode expansions (2.21) and (3.13) it is straightforward to obtain the full effective $4 \mathrm{~d}$ action from the $6 \mathrm{~d}$ action (2.9). The result reads

$$
\begin{aligned}
S_{4}= & \int d^{4} x\left(-\frac{1}{4} F_{0}^{\mu \nu} F_{0 \mu \nu}-\partial^{\mu} \bar{\varphi}_{0} \partial_{\mu} \varphi_{0}-\frac{1}{2} f^{2}\right. \\
& +\sum_{l, m}\left(-\frac{1}{4} F_{-l,-m}^{\mu \nu} F_{\mu \nu, l, m}+\frac{1}{2} \bar{M}_{-l,-m} M_{l, m} A_{-l,-m}^{\mu} A_{\mu, l, m}\right. \\
& -\partial^{\mu} \bar{\varphi}_{l, m} \partial_{\mu} \varphi_{l, m}-\frac{1}{4}\left|M_{-l,-m} \bar{\varphi}_{-l,-m}+\bar{M}_{l, m} \varphi_{l, m}\right|^{2} \\
& \left.-\frac{i}{\sqrt{2}} A_{-l,-m}^{\mu} \partial_{\mu}\left(M_{-l,-m} \bar{\varphi}_{-l,-m}-\bar{M}_{l, m} \varphi_{l, m}\right)\right) \\
& +\sum_{n, j}\left(-i \psi_{n, j} \sigma^{\mu} \bar{D}_{\mu} \bar{\psi}_{n, j}-i \chi_{n, j} \sigma^{\mu} D_{\mu} \bar{\chi}_{n, j}\right. \\
& -\sqrt{2 q f(n+1)} \chi_{n, j} \psi_{n+1, j}-\sqrt{2} q \varphi_{0} \chi_{n, j} \psi_{n, j} \\
& \left.-\sqrt{2 q f(n+1)} \bar{\chi}_{n, j} \bar{\psi}_{n+1, j}-\sqrt{2} q \bar{\varphi}_{0} \bar{\chi}_{n, j} \bar{\psi}_{n, j}\right) \\
& +\sum_{l, m ; n, j ; n^{\prime}, j^{\prime}} C_{n, j ; n^{\prime}, j^{\prime}}^{l, m}\left(-q \psi_{n^{\prime}, j^{\prime}} \sigma^{\mu} A_{\mu, l, m} \bar{\psi}_{n, j}+q \chi_{n, j} \sigma^{\mu} A_{\mu, l, m} \bar{\chi}_{n^{\prime}, j^{\prime}}\right. \\
& \left.\left.-\sqrt{2} q \varphi_{l, m} \chi_{n, j} \psi_{n^{\prime}, j^{\prime}}-\sqrt{2} q \bar{\varphi}_{-l,-m} \bar{\chi}_{n^{\prime}, j^{\prime}} \bar{\psi}_{n, j}\right)\right) .
\end{aligned}
$$

Here the covariant derivatives $D_{\mu}, \bar{D}_{\mu}$ only involve the zero mode $A_{0 \mu}$ of the gauge field, and the cubic couplings of the gauge and matter KK modes are given by the overlap integrals

$$
C_{n, j ; n^{\prime}, j^{\prime}}^{l, m}=\int_{T_{2}} d^{2} x \lambda_{l, m} \bar{\xi}_{n, j} \xi_{n^{\prime}, j^{\prime}}
$$

The action (3.19) describes the gauge modes $\varphi_{0}, \varphi_{l, m}, A_{0 \mu}, A_{\mu, l, m}$ and the fermion modes $\psi_{n, j}, \chi_{n, j}$ as well as their interactions. In unitary gauge the mixing between $A_{l, m}^{\mu}$ and one linear combination of $\varphi_{l, m}$ and $\bar{\varphi}_{l, m}$ is eliminated whereas the orthogonal combination describes a tower of real, massive scalars. ${ }^{7}$

\footnotetext{
${ }^{7}$ In the supersymmetric case this was discussed in [18].
} 
The mode functions of the charged matter fields are related by creation or annihilation operators, for instance (cf. (2.14), (2.19)),

$$
\bar{\xi}_{n-1, j}=-\frac{i}{\sqrt{n}} a_{-} \bar{\xi}_{n, j}=\frac{1}{\sqrt{2 n q f}}\left(\partial_{\bar{z}}+q f z\right) \bar{\xi}_{n, j} .
$$

Using expressions of this kind and performing partial integrations one easily derives the following relations between the cubic couplings:

$$
\begin{aligned}
\sqrt{2 q f}\left(\sqrt{n} C_{n-1, j ; n^{\prime}, j^{\prime}}^{l, m}-\sqrt{n^{\prime}+1} C_{n, j ; n^{\prime}+1, j^{\prime}}^{l, m}\right) & =\bar{M}_{l, m} C_{n, j ; n^{\prime}, j^{\prime}}^{l, m}, \\
\sqrt{2 q f}\left(-\sqrt{n+1} C_{n+1, j ; n^{\prime}, j^{\prime}}^{l, m}+\sqrt{n^{\prime}} C_{n, j ; n^{\prime}-1, j^{\prime}}^{l, m}\right) & =-M_{l, m} C_{n, j ; n^{\prime}, j^{\prime}}^{l, m}
\end{aligned}
$$

With these relations it is straightforward to prove the invariance of the action (3.19) under the transformations (3.5), (3.7), (3.12), (3.17) and (3.18). For instance, for the Yukawa interactions one has

$$
\begin{aligned}
\delta & \sum_{l, m ; n, j ; n^{\prime}, j^{\prime}} C_{n, j ; n^{\prime}, j^{\prime}}^{l, m} \varphi_{l, m} \chi_{n, j} \psi_{n^{\prime}, j^{\prime}} \\
= & \left(-\left(\bar{\epsilon} \bar{M}_{l, m}-\epsilon M_{l, m}\right) C_{n, j ; n^{\prime}, j^{\prime}}^{l, m}\right. \\
& +\sqrt{2 q f}\left(-\epsilon \sqrt{n+1} C_{n+1, j ; n^{\prime}, j^{\prime}}^{l, m}+\bar{\epsilon} \sqrt{n} C_{n-1, j ; n^{\prime}, j^{\prime}}^{l, m}\right) \\
& \left.-\sqrt{2 q f}\left(\epsilon \sqrt{n^{\prime}} C_{n, j ; n^{\prime}-1, j^{\prime}}^{l, m}+\bar{\epsilon} \sqrt{n^{\prime}+1} C_{n, j ; n^{\prime}+1, j^{\prime}}^{l, m}\right)\right) \varphi_{l, m} \chi_{n, j} \psi_{n^{\prime}, j^{\prime}} \\
= & \left(-\bar{\epsilon}\left(\bar{M}_{l, m} C_{n, j ; n^{\prime}, j^{\prime}}^{l, m}+\sqrt{2 q f}\left(\sqrt{n} C_{n-1, j ; n^{\prime}, j^{\prime}}^{l, m}-\sqrt{n^{\prime}+1} C_{n, j ; n^{\prime}+1, j^{\prime}}^{l, m}\right)\right)\right. \\
& +\epsilon\left(M_{l, m} C_{n, j ; n^{\prime}, j^{\prime}}^{l, m}-\sqrt{2 q f}\left(\sqrt{n+1} C_{n+1, j ; n^{\prime}, j^{\prime}}^{l, m}-\sqrt{n^{\prime}} C_{n, j ; n^{\prime}-1, j^{\prime}}^{l, m}\right)\right) \\
& \times \varphi_{l, m} \chi_{n, j} \psi_{n^{\prime}, j^{\prime}} .
\end{aligned}
$$

Using eqs. (3.22) one finds

$$
\delta \sum_{l, m ; n, j ; n^{\prime}, j^{\prime}} C_{n, j ; n^{\prime}, j^{\prime}}^{l, m} \varphi_{l, m} \chi_{n, j} \psi_{n^{\prime}, j^{\prime}}=0 .
$$

In the same way one shows the invariance of the other cubic and bilinear terms in the action (3.19) involving gauge KK modes. For the invariance of the remaining terms, which was already demonstrated above, the shift (3.12) of the zero mode is crucial, $\delta \varphi_{0}=\sqrt{2} \bar{\epsilon} f$.

It is instructive to contrast this result with the generation of a mass for the WL scalar in gauge-Higgs unification without flux. The inverse size of the torus plays the role of a cutoff, and a discrete symmetry, a remnant of the gauge symmetry in the compact dimensions, keeps the mass finite. Explicit expressions for the effective potential of the WL scalar have been obtained in refs. [14, 29]. For a square torus of size $L=2 \pi R$ one obtains [30]

$$
m_{\varphi_{0}}^{2} \approx 0.19 \times \frac{\alpha}{\pi} \frac{1}{R^{2}}
$$

As expected, the result is the product of a loop factor and the cutoff. Turning on flux changes the situation drastically. The full $4 \mathrm{~d}$ action including the couplings of all KK modes 
is invariant with respect to a symmetry under which the scalar $\varphi_{0}$ shifts by a constant. The origin of this symmetry is the shift of $\varphi$ under translations on the torus, which compensates for the spontaneous breaking of translation invariance by the flux potential. As a result, the mass of the WL scalar vanishes.

\section{Summary and outlook}

Motivated by the hierarchy problem of the electroweak theory we have studied the effect of magnetic flux on quantum corrections to a scalar mass term in a model of gauge-Higgs unification. We considered the simplest possible example, a $6 \mathrm{~d}$ Weyl fermion with Abelian gauge interaction, compactified on a torus.

We first analyzed the symmetries of the $6 \mathrm{~d}$ theory on a torus. In the presence of the background gauge field translational invariance is realized non-linearly, and the Higgs field transforms with a shift. Moreover, the theory has a well known local 6d symmetry under which the Higgs field also transforms with a shift, and which changes the boundary conditions of the charged fields. Using the familiar harmonic oscillator algebra a complete orthonormal set of mode functions was constructed for the two 4d Weyl fermions with opposite charge, which are contained in the $6 \mathrm{~d}$ Weyl fermion. The Higgs field and the vector field have the standard Kaluza-Klein mode expansion. The effective $4 \mathrm{~d}$ action contains as zero modes a multiplicity of Weyl fermions, determined by the magnetic flux, and a complex scalar with chiral couplings to pairs of different fermions.

Quantum corrections were studied in three steps. Keeping just the lowest lying massive Dirac fermion, a quadratic divergence for the scalar mass term is generated at one loop, as expected. However, once the full tower of massive states is included, the total correction to the scalar mass term vanishes, confirming our earlier result. The origin of this cancellation of quantum corrections is a symmetry of the $4 \mathrm{~d}$ effective action. Starting from the two symmetries of the $6 \mathrm{~d}$ theory, translations and gauge invariance, we identified a transformation law of the $4 \mathrm{~d}$ fields which leaves the $4 \mathrm{~d}$ action invariant. Under this transformation the complex scalar transforms with a shift, which prevents the generation of a mass term.

In a third step we generalized this result to the complete $4 \mathrm{~d}$ theory, including the Kaluza-Klein excitations of scalar and vector fields. Using properties of the mode functions we obtained relations among the cubic couplings, which allowed us to demonstrate that the full $4 \mathrm{~d}$ theory has an exact symmetry under which the complex Higgs field transforms with a shift. The origin of this shift symmetry are the translation symmetries of the torus. Assuming a renormalization scheme, which preserves this symmetry, we conclude that no scalar mass term will be generated at any loop order. This is the main result of the present paper.

What is the relevance of this result for the hierarchy problem of the electroweak theory? The effective low energy action of our model was given in section 2 . The action has a shift symmetry, and a vacuum expectation value of the scalar field does not generate mass terms for chiral fermions, which is the key feature of the Standard Model. In order to obtain more realistic low energy models one has to consider non-Abelian gauge theories in 
higher dimensions. It is then possible to obtain flux compactifications with gauge-Higgs unification where the Higgs field couples to chiral fermions and all fields have Landau-level excitations. It remains to be seen whether also in these theories, where the Higgs field is charged, an approximate shift symmetry can be realized once the contribution of KaluzaKlein excitations is taken into account. These questions are currently under investigation.

\section{Acknowledgments}

We thank Constantin Bachas, Arthur Hebecker, Miguel Montero, and Augusto Sagnotti for valuable discussions. This work was supported by the German Science Foundation (DFG) within the Collaborative Research Center (SFB) 676 "Particles, Strings and the Early Universe". M.D.'s work is part of the D-ITP consortium, a program of the Netherlands Organisation for Scientific Research (NWO) that is funded by the Dutch Ministry of Education, Culture and Science (OCW). E.D. was supported in part by the "Agence Nationale de la Recherche" (ANR).

\section{A Quantum mechanics on the magnetized torus}

It is possible to understand the properties under translations on the magnetized torus by using elementary quantum mechanics arguments (see e.g. [31, 32]). In the presence of a magnetic field, $F_{56}=f$, the gauge potential in the two directions of the torus, in the symmetric gauge we used in the previous sections, is given by

$$
A_{5}=-\frac{1}{2} f x_{6}+a_{5}, \quad A_{6}=\frac{1}{2} f x_{5}+a_{6},
$$

where $a_{5,6}$ are constants (i.e. Wilson lines). The non-trivial transformations of the gauge potentials under translations along the two cycles of the torus are

$$
A_{5}\left(x_{5}, x_{6}+\epsilon_{6}\right)=A_{5}\left(x_{5}, x_{6}\right)-\frac{1}{2} f \epsilon_{6}, \quad A_{6}\left(x_{5}+\epsilon_{5}, x_{6}\right)=A_{6}\left(x_{5}, x_{6}\right)+\frac{1}{2} f \epsilon_{5},
$$

which can also be described as gauge transformations with parameters,

$$
\Lambda_{5}=\Lambda_{5}^{(0)}+\frac{1}{2} f \epsilon_{5} x_{6}, \quad \Lambda_{6}=\Lambda_{6}^{(0)}-\frac{1}{2} f \epsilon_{6} x_{5}
$$

where $\Lambda_{5,6}^{(0)}$ describe constant gauge transformations which allow us to eliminate trivial (by constant factors) transformations of the wave functions. The Hamiltonian $H$ of a particle of charge $q$ on the magnetized torus is given by

$$
H=\frac{1}{2}\left(P_{5}-\frac{q f}{2} x_{6}+q a_{5}\right)^{2}+\frac{1}{2}\left(P_{6}+\frac{q f}{2} x_{5}+q a_{6}\right)^{2},
$$

where $P_{5}, P_{6}$ are the momenta, acting on the wave functions in the standard way $P_{5,6}=$ $-i \partial_{5,6}$. The operators related to translations by $\epsilon_{5,6}$ on the torus in a flux background have to commute with the Hamiltonian (A.4). They are explicitly given by

$$
\Pi_{5}=e^{i \epsilon_{5}\left(P_{5}+\frac{q f}{2} x_{6}\right)}, \quad \Pi_{6}=e^{i \epsilon_{6}\left(P_{6}-\frac{q f}{2} x_{5}\right)} .
$$


Since they commute with $H$ one can choose wave functions which are eigenvectors of the translations operators. From (A.5) or (A.3) one can derive the transformation behavior of wave functions of charged fields under lattice translations

$$
\begin{aligned}
& \Psi\left(x_{5}+L, x_{6}\right)=e^{-\frac{i}{2} q f L x_{6}+i q L a_{5}} \Psi\left(x_{5}, x_{6}\right), \\
& \Psi\left(x_{5}, x_{6}+L\right)=e^{\frac{i}{2} q f L x_{5}+i q L a_{6}} \Psi\left(x_{5}, x_{6}\right) .
\end{aligned}
$$

Notice that by taking closed loops around the two cycles, $\epsilon_{5,6}=L$, and imposing singlevaluedness of the wave function, one can derive the quantization of the magnetic flux $[5,31]$. By defining complex translations with

$$
\epsilon=\frac{1}{2}\left(\epsilon_{5}+i \epsilon_{6}\right)
$$

one obtains a complex translation operator on the torus implementing $z \rightarrow z+\epsilon$. Using the Campbell-Hausdorff formula one finds

$$
\begin{aligned}
\Pi_{\epsilon}=\Pi_{6} \Pi_{5} & =\exp \left(\epsilon \partial_{z}+\bar{\epsilon} \bar{\partial}_{z}-q f(\epsilon \bar{z}-\bar{\epsilon} z)-\frac{q f}{2}\left(\epsilon^{2}-\bar{\epsilon}^{2}\right)\right) \\
& =\exp \left(-i \sqrt{2 q f}\left(\epsilon a_{-}^{\dagger}+\bar{\epsilon} a_{-}\right)-\frac{q f}{2}\left(\epsilon^{2}-\bar{\epsilon}^{2}\right)\right)
\end{aligned}
$$

with $a_{-}$and $a_{-}^{\dagger}$ defined in eqs. (2.15). This symmetry of the Hamiltonian is a standard one since, as we will discuss below, $a_{-}$and $a_{-}^{\dagger}$ are acting in the degenerate Fock space of Landau levels of a given mass, creating the degeneracy described by the quantum number $j$.

On the other hand, the Schrödinger equation of a charged particle in the magnetic field has another, less obvious symmetry. Let us search for a symmetry of the Schrödinger equation,

$$
H \Psi=E \Psi, \quad H^{\prime} \Psi^{\prime}=E \Psi^{\prime},
$$

which mixes states of different mass. It is possible to realize this, while keeping the energy eigenvalue $E$ invariant, if one also performs changes of the field $\varphi$. First of all, the Hamiltonian (A.4) can be written as

$$
H=q f\left(a_{+}^{\dagger} a_{+}+\frac{1}{2}\right)+i q \sqrt{q f}\left(\varphi a_{+}^{\dagger}-\bar{\varphi} a_{+}\right)+q^{2}|\varphi|^{2},
$$

with $a_{+}$and $a_{+}^{\dagger}$ defined in eqs. (2.14). A symmetry of the Schrödinger equation is of the form

$$
\Psi^{\prime}=U \Psi, \quad H^{\prime}=U H U^{-1},
$$

with a unitary operator $U$. For an infinitesimal transformation $U=e^{i T} \simeq 1+i T$, with $T$ a hermitian generator, one finds

$$
\delta \Psi=i T \Psi, \quad \delta H=i[T, H]
$$


where in our case

$$
\delta H=i q \sqrt{q f}\left(\delta \varphi a_{+}^{\dagger}-\delta \bar{\varphi} a_{+}\right)+q^{2}(\varphi \delta \bar{\varphi}+\bar{\varphi} \delta \varphi) .
$$

It is then straightforward to verify that (A.12) is satisfied, with

$$
\begin{aligned}
T & =-\sqrt{2 q f}\left(\epsilon a_{+}+\bar{\epsilon} a_{+}^{\dagger}\right), \\
\delta \varphi & =\sqrt{2} \bar{\epsilon} f .
\end{aligned}
$$

Finally, the hidden and non-linearly realized symmetry of the Schrödinger equation acts as

$$
\begin{aligned}
\delta \Psi & =-i \sqrt{2 q f}\left(\epsilon a_{+}+\bar{\epsilon} a_{+}^{\dagger}\right) \Psi \\
\delta \varphi & =\sqrt{2} \bar{\epsilon} f .
\end{aligned}
$$

This is the quantum mechanical analog of the higher-dimensional symmetry found in field theory, mixing all Landau mass levels, under which the scalar $\varphi$ transforms as a Goldstone boson, see eqs. (3.4), (3.6) and (3.12). In the quantum mechanical case, in which the gauge field is external (not quantized), this would just imply that the gauge potential is unphysical and can be set to zero.

Finally, we would like to briefly discuss the properties of wave functions in the magnetic field in the symmetric gauge that we are using (see, for example [32]). One can introduce an angular momentum operator ${ }^{8}$

$$
-J=x_{5} P_{6}-x_{6} P_{5}=z \partial_{z}-\bar{z} \partial_{\bar{z}}=a_{+}^{\dagger} a_{+}-a_{-}^{\dagger} a_{-} .
$$

Notice that $\left[H_{0}, J\right]=0$ and $\left[J, \Pi_{\epsilon}\right] \neq 0$, where $H_{0}=H(\varphi=0)$. Hence, one can choose wave functions with definite angular momentum or eigenvectors of the translation operator, but in general not both simultaneously. The usual choice are states of definite angular momentum $j$. In this case, $a_{-}, a_{-}^{\dagger}$ generate the Fock space of states $|n, j\rangle, j=0, \ldots, N-1$ for a given oscillator quantum number $n$, where $N$ is the magnetic field flux. Indeed, notice in particular that, in non-compact space,

$$
|0, j\rangle=\frac{\left(a_{-}^{\dagger}\right)^{j}}{\sqrt{j !}}|0,0\rangle \sim \bar{z}^{j} e^{-q f|z|^{2}},
$$

with corresponding wave functions in the compact space constructed by adding images, as usual. The Fock space of wave functions can be explicitly constructed according to

$$
|n, j\rangle=\frac{\left(a_{+}^{\dagger}\right)^{n}}{\sqrt{n !}} \frac{\left(a_{-}^{\dagger}\right)^{j}}{\sqrt{j !}}|0,0\rangle .
$$

Open Access. This article is distributed under the terms of the Creative Commons Attribution License (CC-BY 4.0), which permits any use, distribution and reproduction in any medium, provided the original author(s) and source are credited.

\footnotetext{
${ }^{8}$ The negative sign in its definition is a matter of convention.
} 


\section{References}

[1] C. Angelantonj and A. Sagnotti, Open strings, Phys. Rept. 371 (2002) 1 [hep-th/0204089] [INSPIRE].

[2] R. Blumenhagen, B. Körs, D. Lüst and S. Stieberger, Four-dimensional String Compactifications with D-branes, Orientifolds and Fluxes, Phys. Rept. 445 (2007) 1 [hep-th/0610327] [INSPIRE].

[3] L.E. Ibanez and A.M. Uranga, String theory and particle physics: An introduction to string phenomenology, Cambridge University Press, Cambridge U.K. (2012).

[4] E. Witten, Some Properties of O(32) Superstrings, Phys. Lett. B 149 (1984) 351.

[5] C. Bachas, A way to break supersymmetry, hep-th/9503030 [INSPIRE].

[6] D. Cremades, L.E. Ibáñez and F. Marchesano, Computing Yukawa couplings from magnetized extra dimensions, JHEP 05 (2004) 079 [hep-th/0404229] [INSPIRE].

[7] T. Kobayashi, R. Maruyama, M. Murata, H. Ohki and M. Sakai, Three-generation Models from $E_{8}$ Magnetized Extra Dimensional Theory, JHEP 05 (2010) 050 [arXiv:1002.2828] [INSPIRE].

[8] Y. Hamada and T. Kobayashi, Massive Modes in Magnetized Brane Models, Prog. Theor. Phys. 128 (2012) 903 [arXiv: 1207.6867] [INSPIRE].

[9] W. Buchmüller, M. Dierigl, F. Ruehle and J. Schweizer, Split symmetries, Phys. Lett. B 750 (2015) 615 [arXiv:1507.06819] [INSPIRE].

[10] Y. Hosotani, Dynamical Mass Generation by Compact Extra Dimensions, Phys. Lett. B 126 (1983) 309.

[11] H. Hatanaka, T. Inami and C.S. Lim, The Gauge hierarchy problem and higher dimensional gauge theories, Mod. Phys. Lett. A 13 (1998) 2601 [hep-th/9805067] [InSPIRE].

[12] G.R. Dvali, S. Randjbar-Daemi and R. Tabbash, The Origin of spontaneous symmetry breaking in theories with large extra dimensions, Phys. Rev. D 65 (2002) 064021 [hep-ph/0102307] [INSPIRE].

[13] N. Arkani-Hamed, A.G. Cohen and H. Georgi, Electroweak symmetry breaking from dimensional deconstruction, Phys. Lett. B 513 (2001) 232 [hep-ph/0105239] [INSPIRE].

[14] I. Antoniadis, K. Benakli and M. Quirós, Finite Higgs mass without supersymmetry, New J. Phys. 3 (2001) 20 [hep-th/0108005] [INSPIRE].

[15] J. Alfaro, A. Broncano, M.B. Gavela, S. Rigolin and M. Salvatori, Phenomenology of symmetry breaking from extra dimensions, JHEP 01 (2007) 005 [hep-ph/0606070] [INSPIRE].

[16] R.D. Peccei and H.R. Quinn, CP Conservation in the Presence of Instantons, Phys. Rev. Lett. 38 (1977) 1440 [INSPIRE].

[17] G. Panico and A. Wulzer, The Composite Nambu-Goldstone Higgs, Lect. Notes Phys. 913 (2016) 1 [arXiv:1506.01961] [INSPIRE].

[18] W. Buchmüller, M. Dierigl, E. Dudas and J. Schweizer, Effective field theory for magnetic compactifications, JHEP 04 (2017) 052 [arXiv: 1611.03798] [INSPIRE].

[19] D.M. Ghilencea and H.M. Lee, Wilson lines and UV sensitivity in magnetic compactifications, JHEP 06 (2017) 039 [arXiv: 1703.10418] [INSPIRE]. 
[20] C. Angelantonj, I. Antoniadis, E. Dudas and A. Sagnotti, Type I strings on magnetized orbifolds and brane transmutation, Phys. Lett. B 489 (2000) 223 [hep-th/0007090] [INSPIRE].

[21] M. Berkooz, M.R. Douglas and R.G. Leigh, Branes intersecting at angles, Nucl. Phys. B 480 (1996) 265 [hep-th/9606139] [INSPIRE].

[22] R. Blumenhagen, L. Görlich, B. Körs and D. Lüst, Noncommutative compactifications of type-I strings on tori with magnetic background flux, JHEP 10 (2000) 006 [hep-th/0007024] [INSPIRE].

[23] G. Aldazabal, S. Franco, L.E. Ibáñez, R. Rabadán and A.M. Uranga, Intersecting brane worlds, JHEP 02 (2001) 047 [hep-ph/0011132] [INSPIRE].

[24] E. Dudas, G. Pradisi, M. Nicolosi and A. Sagnotti, On tadpoles and vacuum redefinitions in string theory, Nucl. Phys. B 708 (2005) 3 [hep-th/0410101] [INSPIRE].

[25] J. Wess and J. Bagger, Supersymmetry and supergravity, Princeton University Press, Princeton U.S.A. (1992).

[26] J. Scherk and J. H. Schwarz, Spontaneous Breaking of Supersymmetry Through Dimensional Reduction, Phys. Lett. B 82 (1979) 60.

[27] A.P. Braun, A. Hebecker and M. Trapletti, Flux Stabilization in 6 Dimensions: D-terms and Loop Corrections, JHEP 02 (2007) 015 [hep-th/0611102] [INSPIRE].

[28] W. Buchmüller, M. Dierigl, F. Ruehle and J. Schweizer, Chiral fermions and anomaly cancellation on orbifolds with Wilson lines and flux, Phys. Rev. D 92 (2015) 105031 [arXiv: 1506.05771] [INSPIRE].

[29] D.M. Ghilencea, D. Hoover, C.P. Burgess and F. Quevedo, Casimir energies for $6 D$ supergravities compactified on $T(2) / Z(N)$ with Wilson lines, JHEP 09 (2005) 050 [hep-th/0506164] [INSPIRE].

[30] M. Dierigl, Aspects of Six-Dimensional Flux Compactifications, Ph.D. Thesis, University of Hamburg, Hamburg Germany (2017).

[31] A. Abouelsaood, C.G. Callan Jr., C.R. Nappi and S.A. Yost, Open Strings in Background Gauge Fields, Nucl. Phys. B 280 (1987) 599 [InSPIRE].

[32] D. Tong, Lectures on the Quantum Hall Effect, arXiv:1606.06687 [INSPIRE]. 